\title{
Recovery of extinction responding in rats following discontinuation of reinforcement of alternative behavior: A test of two explanations
}

\author{
RICHARD A. RAWSON, HAROLD LEITENBERG, JAMES A. MULICK \\ and MARK F. LEFEBVRE \\ University of Vermont, Burlington, Vermont 05401
}

\begin{abstract}
One procedure which has been used to supplement extinction in order to produce faster and more complete response suppression is to provide reinforcement for some alternative response which is incompatible with the response undergoing extinction. When reinforcement for the alternative behavior is discontinued, however, substantial recovery of the original response has often been observed. The present set of experiments demonstrated that such recovery is best accounted for by a "response prevention" hypothesis rather than by a "discriminative cue" hypothesis. High-frequency reinforcement of alternative behavior during the first half of an extinction phase seems similar in effect to procedures which physically prevent rats from emitting the response programmed for extinction.
\end{abstract}

Two procedures which have been widely used to produce rapid response suppression are punishment of a response being extinguished and reinforcement for an alternative response incompatible with a response undergoing extinction. Although both procedures augment the suppression produced by extinction, their long-term effectiveness relative to extinction alone is questionable. For example, discontinuation of punishment often results in some recovery of the response, even though extinction is still in effect for that response (Estes, 1944, Experiment 1; Rawson \& Leitenberg, 1973; Skinner, 1938) and, when the reinforcement for an alternative behavior is discontinued, there is partial recovery of the original behavior (Enkema, Slavin, Spaeth, \& Neuringer, 1972; Leitenberg, Rawson, \& Bath, 1970; Leitenberg, Rawson, \& Mulick, 1975; Rawson \& Leitenberg, 1973). Although there are methods for producing more permanent suppression using these techniques, e.g., the use of high-intensity shock in the punishment situation (Boe \& Church, 1967) or reinforcing an alternative response for an extended period of time (Leitenberg et al., 1975, Experiment 4), there is sufficient evidence to expect substantial response recovery when these procedures are used for a brief period and then discontinued.

This paper is based on a portion of the research contained in the senior author's dissertation submitted to the Department of Psychology, University of Vermont, in partial fulfillment of the requirements for the $\mathrm{PhD}$ degree. $\mathrm{R}$. A. Rawson is now at the H.A.L.T. Project, Oxnard, California; J. A. Mulick is now at Murdoch Center, Butner, North Carolina. Reprint requests should be sent to Richard A. Rawson, PhD, H.A.L.T. Project, 522 North A Street, Oxnard, California 93030.
Two hypotheses have been proposed to account for response recovery when punishment is discontinued in a combined punishment-extinction technique. The first maintains that when punishment and extinction are used in combination, the aversive properties of the punisher suppress the frequency of the punished response and thereby prevent the response from undergoing extinction (Estes, 1944; Skinner, 1938). In a sense, the punishment is discontinued, the response once again becomes "available" and the necessary nonreinforced responses are made which produce permanent suppression of the response. Implicit in this explanation is the concept that a response can only be permanently weakened by the repeated occurrence of that response followed by nonreinforcement. The second hypothesis maintains that response recovery can be attributed to the discriminative properties of the punisher (Azrin \& Holz, 1966). Specifically, when punishment and extinction are introduced simultaneously, the punishing stimulus may become a discriminative cue for extinction conditions. Subsequent discontinuation of punishment may signal that extinction is over, resulting in resumption of the response until extinction takes place under the new stimulus conditions.

Although the two hypotheses just described plausibly account for recovery of extinction responding following discontinuation of punishment, there has been no similar attempt to explain recovery from suppression when reinforcement for an alternative response is discontinued. One possible explanation for this phenomenon is similar to the Estes (1944) interpretation of recovery from punishment. Since 
studies on the effects of reinforcing an alternative response have typically used a very dense schedule of reinforcement for the new behavior, the animals quickly switch from the old behavior which is no longer reinforced (Enkema et al., 1972; Leitenberg et al., 1970; Rawson \& Leitenberg, 1973). In doing so, the animals get very little experience in making the old response without reinforcement, which may "prevent" extinction of the original behavior from occurring. When reinforcement for the alternative response is discontinued, the original response once again becomes "available" and the necessary extinction responses occur.

The second hypothesis parallels the Azrin and Holz (1966) recovery from punishment hypothesis. Since extinction conditions for the original response and reinforcement for the alternative response have been introduced simultaneously, reinforcement for responses on the alternative lever possibly serve as discriminative cues for extinction conditions for the original response (Leitenberg et al., 1970, 1975). When reinforcement for the alternative response is subsequently discontinued, it may signal that baseline conditions are back in effect, resulting in recovery of the original behavior.

This paper presents experiments designed to evaluate whether either of the hypothesis presented above can adequately account for the response recovery which occurs following discontinuaton of reinforcement for an alternative response.

\section{EXPERIMENT 1}

Providing reinforcement for a new behavior during extinction of an old behavior may be functionally equivalent to physically interfering with the emission of the old behavior. According to this hypothesis, withdrawal of reinforcement for the alternative behavior might produce effects similar to those observed if such physical restraint were removed. The purpose of this experiment is to compare similar periods of standard extinction, extinction plus reinforcement of an alternative response, and two physical response prevention procedures. In one procedure, the subjects are placed in the environment in which they previously made the original response, but the response is physically unavailable. This group is referred to as the "response prevention" group. The second procedure is similar to that used by Skinner $(1938,1950)$ in his work on forgetting. Subjects in this group are simply not placed in the experimental chamber during the second phase of the experiment. This group is referred to as the "forgetting group." If reinforcement of an alternative response produces functional response prevention, the discontinuation of reinforcement for the alternative behavior should result in an amount of recovery similar to that which occurs when the physical response prevention treatments are discontinued.

\section{Method}

Subjects. The subjects were 40 experimentally naive, male hooded rats purchased from the Canadian Breeding Laboratory. The animals, which were 100-120 days old at the beginning of the experiment, were maintained at $80 \%$ of their ad-lib weights on approximately $11 \mathrm{~g}$ of food per day.

Apparatus. Two operant chambers enclosed in soundattenuating cubicles were used. Each $25 \times 18 \times 15 \mathrm{~cm}$ chamber was equipped with a grid floor, a food cup, two identical response levers, one of which was mounted to a Lehigh Valley retractable unit (Model 1407), and jewel lamp $5 \mathrm{~cm}$ above each lever. Each lever was mounted $7.6 \mathrm{~cm}$ above the floor on the same end of the chamber. The right-hand lever (Lever A) was mounted $2.5 \mathrm{~cm}$ from the right wall; the left-hand lever (Lever B) was mounted $2.5 \mathrm{~cm}$ from the left wall. The food cup was approximately halfway between the levers, $2.5 \mathrm{~cm}$ from the floor. Reinforcement consisted of a single $45-\mathrm{mg}$ Noyes pellet delivered automatically via a Scientific Prototype Model D700 dispenser. A Foringer audio generator (Model AU 902) was used to produce a continuous $70 \mathrm{~dB}$ (re $20 \mu \mathrm{N} / \mathrm{m}^{2}$ ) white masking noise. Exhaust fans mounted on the outside of each cubicle provided ventilation as well as additional masking noise.

Procedure. After all subjects had been shaped to press Lever A and given one $30-\mathrm{min}$ pretraining session of variable interval (VI) 30-sec (range: 5-300 sec) reinforcement for Lever A responding, Phase 1 of the experiment began. This phase consisted of five 30-min sessions during which Lever $\mathrm{A}$ responding was reinforced on a VI 30-sec schedule. During Phase 1, the training phase, only Lever A was present in the chamber.

Following Phase 1, subjects were randomly assigned to one of four groups. One group was a standard extinction control group. During the five 30 -min sessions of Phase 2, subjects in this group were placed on extinction for Lever $A$ responses and Lever B was introduced but responses on Lever B were not reinforced. The second group was the standard reinforced alternative response group. Subjects in this group experienced extinction conditions for responses on Lever $A$ while being reinforced for Lever $B$ responses on a fixed ratio (FR) 10 schedule. A 3-sec changeover delay was used to prevent adventitious reinforcement of Lever $A$ responses. An FR 10 schedule was chosen in order 10 rapidly suppress the original response. II is also a schedule employed in previous studies where subsequent recovery was noted.

A third group was physically prevented from making any leverpress responses during Phase 2 . Subjects in this group, the response prevention group, were placed in the chambers for five 30 -min sessions during Phase 2 , but there were no levers present. These subjects were not allowed to experience extinction conditions, since they were physically prevented from making the original response. However, an argument for "latent extinction," i.e., classical extinction to the cues for the chamber associated with reinforcement, could be made. Subjects in the fourth group, the forgetting group, were not placed in the chamber during Phase 2. These subjects were removed from their cages for $30 \mathrm{sec}$ of handling and weighing per day during the 5 days of Phase 2, but did not receive any exposure to the chambers.

During Phase 3, the recovery phase, all subjects were treated identically. This phase consisted of five 30 -min sessions, during which Levers $\mathrm{A}$ and $\mathrm{B}$ were present but there was no reinforcement available for responses on either lever.

\section{Results}

The mean number of responses on Lever $\mathrm{A}$ for each of the four groups across the three phases of the experiment are presented in Figure 1. The mean 


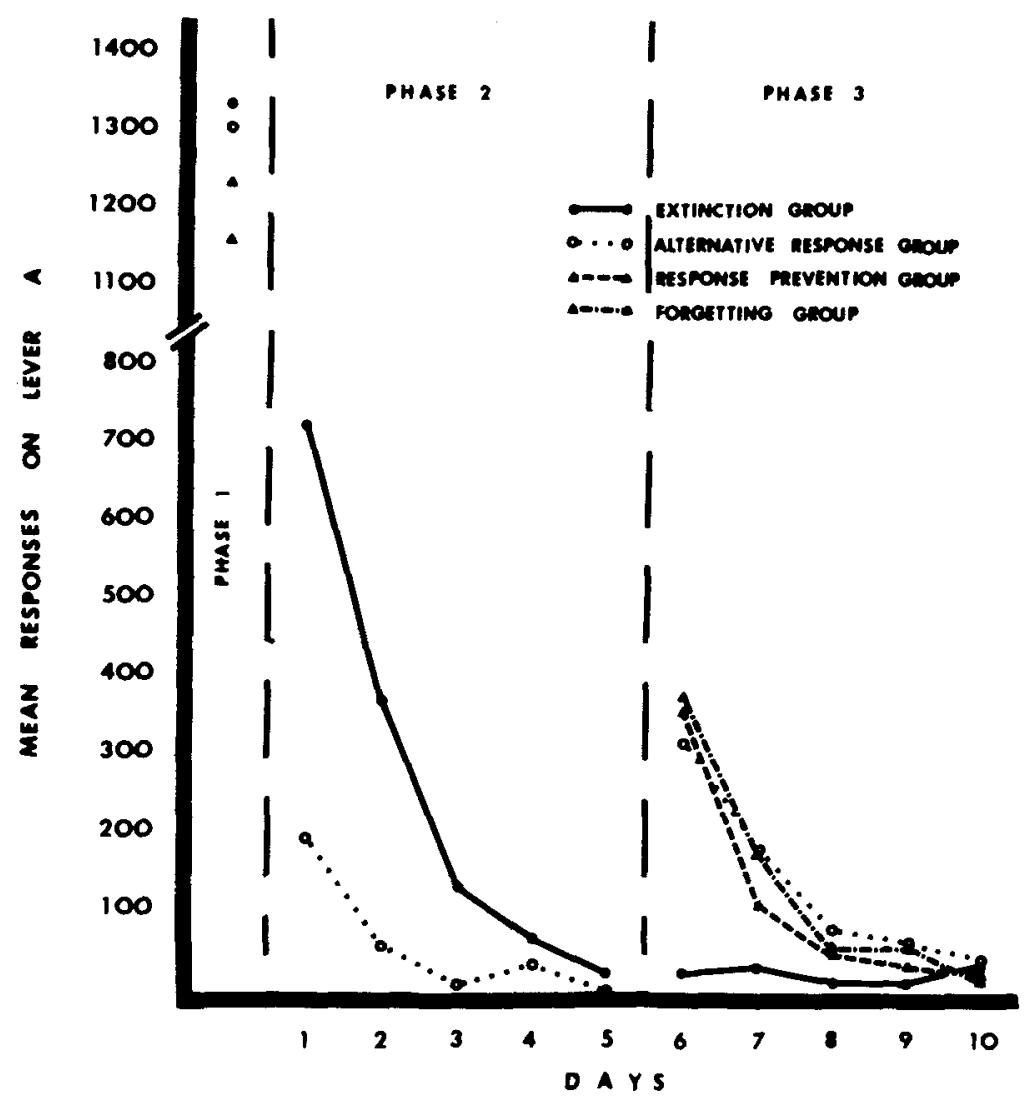

Figure 1. Mean Lever A responses during Phases 1 (training), 2 (extinction), and 3 (recovery) in Experiment 1 .

total Lever A responses during Phase 1 for the four groups was as follows: extinction group, 6,718 ; reinforced alternative response group, 6,563; response prevention group, 6,197; forgetting group, 5,815. A one-way analysis of variance indicated that the Phase 1 response totals were not significantly different $(F<1)$. The mean daily response totals for each group are presented in the left panel in Figure 1.

Figure 1 shows that reinforcement for Lever B responding produced marked suppression of Lever A responding. The mean Lever A response totals during Phase 2 for the extinction group and the reinforced alternative response group were 1,339 and 318 , respectively, a difference that was statistically significant $(t=4.28, p<.001)$. The degree of suppression produced by the reinforced alternative response treatment is similar to that reported in earlier studies (Leitenberg et al., 1970, 1975). Since the other two groups were not allowed to make Lever A responses, there were no data for them in this phase.

Figure 1 also shows that, when reinforcement for Lever B was removed in Phase 3, there was substantial recovery of Lever A responding by the reinforced alternative response group. Furthermore, the response prevention group and the forgetting group made approximately as many responses in Phase $\mathbf{3}$ as the group formerly reinforced for Lever B responding during Phase 2 . The Lever $A$ response totals during this phase were: extinction group, 125; reinforced alternative response group, 702; response prevention group, 596; forgetting group, 704. A oneway analysis of variance of the Phase 3 Lever A totals indicated a significant difference between the groups $[F(3,36)=5.46, p<.01]$. Duncan's Multiple Range tests indicated that the extinction group differed from each of the other three groups during this phase $(\mathrm{p}<.01)$ but that the other three groups did not differ significantly from each other $(p>.10)$.

\section{Discussion}

The response prevention hypothesis predicted that the suppression produced by a reinforced alternative response was due to a type of functional response prevention. If that was the case, the recovery from reinforced alternative response-produced suppression should have been similar to the recovery from a period of "true" response prevention. This experiment demonstrates that the response recovery occurring after a period of suppression produced by a reinforced alternative response is very similar to the recovery from suppression resulting when a 
physical response prevention procedure is discontinued and thus provides substantial support for the response prevention hypothesis. Although the Phase 3 response totals for the subjects in the alternative response group did appear similar to those of the subjects in the forgetting and response prevention groups, it may be that the response recovery resulted from two different processes. The response recovery which resulted from the discontinuation of the forgetting and response prevention procedures clearly represents a recovery from "true" response prevention. However, the recovery which occurred following the discontinuation of the alternative response procedure could have resulted from a "functional response prevention" or from the removal of the discriminative cues for extinction provided by the reinforcement of the alternative behavior. Therefore, although this experiment does support the plausibility of the response prevention hypothesis, it is necessary to test directly the discrimination cue hypothesis in order to assess the adequacy of this latter hypothesis in explaining the recovery from suppression.

\section{EXPERIMENT 2}

Experiment 2 evaluated the discriminative cue explanation for recovery of exinction responding following discontinuation of reinforcement for an alternative response. One way to insure that reinforcement for an alternative behavior does not become a discriminative cue for extinction is to reinforce the alternative behavior not only during the extinction phase but also during the baseline phase in which the original response is reinforced. In this way, reinforcement for the alternative behavior could not become a discriminative cue for extinction, since it would have been paired equally with both reinforcement conditions and extinction conditions for the original behavior. Ferster and Skinner (1957) defined the condition in which two responses are available and reinforced independently as a concurrent schedule. A comparison of groups receiving the standard extinction and extinction plus reinforced-alternative-response procedures (i.e., Leitenberg et al., 1970, 1975) with comparable groups receiving these procedures after having two responses concurrently available in the baseline phase should determine the viability of the discriminative cue hypothesis.

\section{Method}

Subjects. The subjects were 40 experimentally naive, male hooded rats purchased from the Canadian Breeding Laboratory. The animals were maintained in an identical manner to the subjects in Experiment 1.

Apparatus. The apparatus was the same as that used in Experiment 1.
Procedure. During all pretraining sessions, only one lever was present in the chamber at any one time. All rats were "shaped" to press Lever $A$ on a continuous reinforcement schedule and received a single 30 -min session in which Lever A responses were reinforced on a VI 30 -sec schedule identical to the schedule used in Experiment 1. On the following day, half the subjects were randomly selected for the concurrent groups and were given one 30 -min session in which responses on Lever B were reinforced on a VI $30-s e c$ schedule. The remaining subjects comprised the standard treatment groups and were not run on this day. During the final 30-min pretraining session, responding on Lever $A$ was reinforced on a VI 30 -sec schedule for all rats.

Phase 1 of the experiment consisted of five 30-min training sessions for all subjects. Subjects in the standard groups received reinforcement for Lever $A$ responses on a VI 30 -sec schedule. Lever B was not present for subjects in these groups during this phase. Subjects in the concurrent groups received reinforcement for responses on Lever A and Lever B according to identical, independently programmed, VI 30 -sec schedules. During this and all subsequent phases, a $3-\mathrm{sec}$ changeover delay was used to prevent adventitious reinforcement.

Phase 2 of the experiment consisted of five 30-min "extinction" sessions in which reinforcement for Lever A responses was discontinued for all subjects. During this phase, half of the subjects in each group received VI 30 -sec reinforcement for Lever B responses (the alternative response), while for the other half of the subjects. Lever B was present in the chamber but responses on it were not reinforced. This division of the standard treatment and concurrent groups resulted in the following four groups of 10 subjects: standard training-extinction (Group E); standard training-extinction plus reinforced alternative (Group AR); concurrent training-extinction (Group CE); concurrent trainingextinction plus reinforced alternative response (Group CAR). Subjects were always assigned randomly to groups.

Phase 3 consisted of five 30 -min "recovery" sessions in which conditions were identical for all four groups. During this phase, extinction conditions were in effect for all groups for responses on both levers during all five sessions.

\section{Results}

A weighted means analysis of variance (Winer, 1971) was necessary due to the elimination of three subjects. One subject in Group CAR was dropped because of sickness. One subject in Group AR and one subject in Group CAR were dropped because neither subject made more than 10 Lever $B$ responses during Phase 2 . Since these subjects never acquired the alternative behavior, it was felt that they should not be part of an analysis which measured the effect of a reinforced alternative behavior.

During Phase 1, the two groups which received access only to Lever A responded more on Lever A than those groups which were concurrently reinforced on Lever B. The mean number of Lever A responses per day, per group during Phase 1 were: Group E, 869; Group AR, 810; Group CE, 449; Group CAR, 521. A two-way analysis of variance on Phase 1 Lever A totals indicated that there was a significant main effect in the comparison between the two concurrent groups and the two standard groups $[F(1,33)=11.55, \mathrm{p}<.001]$. However, the two concurrent groups did not differ significantly from each other and the two standard groups did not differ 
significantly from each other $(F<1)$. There was no significant interaction.

Following Phase 1, subsequent group comparisons were complicated by the fact that the analysis of variance on the response totals across each phase did not give a good indication of the relative amounts of suppression produced, since the groups did not begin Phase 2 with the same amount of experience on Lever A. Consequently, suppression ratios were used to analyze the response totals on Lever A during subsequent phases. Ratios were computed for each subject during Phase 2 according to the following formula:

\section{Lever A Responses during Phase 2 Lever A Responses during Phases 1 \& 2}

Using this formula, a ratio of 0.5 would indicate that there was no response suppression during Phase 2 , while a ratio of 0.16 would indicate that the subject had made only one-fifth as many Lever A responses during Phase 2 as it had made during Phase 1.

The mean suppression ratios for the four groups during the Phase 2 were as follows: Group E, 0.152; Group AR, 0.085; Group CE, 0.188; Group CAR, 0.142 . An analysis of variance indicated that the two alternative response groups were suppressed to a greater degree than the two extinction groups $[F(1,33)=15.32, p<.01]$. In addition, the two concurrent groups were suppressed significantly less than the two standard groups $[F(1,33)=9.98$, $\mathrm{p}<.05]$. There was no significant interaction $(\mathrm{F}<1)$. Daily ratios were computed for each group on each day of Phases 2 and 3 and are plotted in Figure 2.

In summary, the analysis of the Phase 2 data indicated that the reinforcement for Lever $\mathrm{B}$ response augmented the response reduction effects of extinction.

Since the major focus of this experiment concerned the recovery from the suppression produced by a reinforced alternative response, the data of primary importance are Lever A responding during Phase 3. To compare the relative amounts of Lever A responding in Phase 3, suppression ratios were calculated for each subject and these ratios were then used in an analysis of variance. The ratios were calculated by the following formula:

\section{Lever A Responses during Phase 3 Lever A Responses during Phases 1 \& 3}

Thus a ratio of 0.5 would indicate no difference in total responses in Phases 1 and 3, a ratio of less than 0.5 would indicate fewer responses in Phase 3 than in Phase 1. The mean suppression ratio values for the four groups during Phase 3 were as follows: Group E,

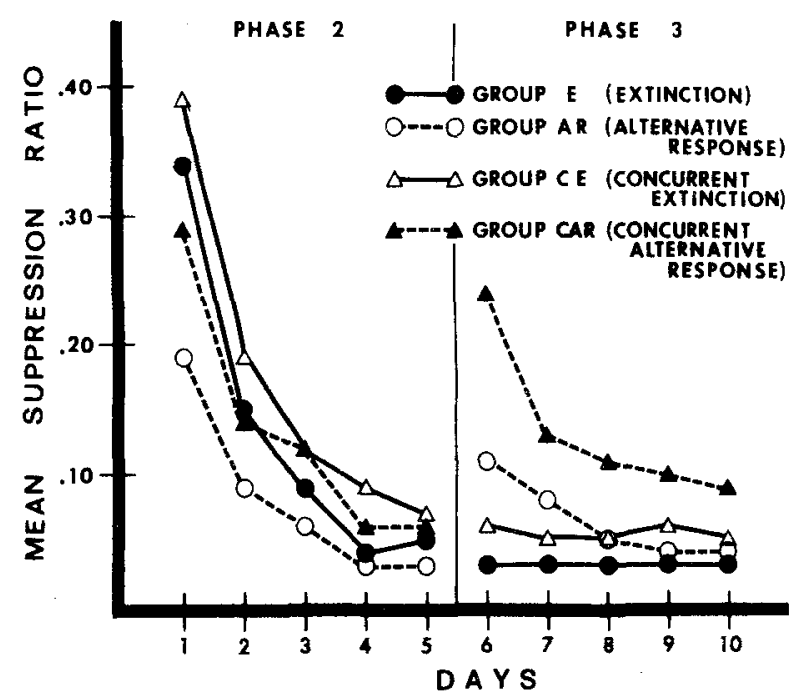

Figure 2. Mean suppression ratios during Phases 2 (extinction) and 3 (recovery) in Experiment 2.

0.030; Group AR, 0.053; Group CE, 0.053; Group CAR, 0.134. The daily pattern of these ratios can be seen in the right panel in Figure 2.

A two-way analysis of variance on these ratio scores indicated that the two alternative response groups' Lever A responding was suppressed significantly less than that of the two extinction groups $[F(1,33)=4.44, p<.05]$. In addition, the two concurrent groups responded significantly more than the two nonconcurrent groups $[\mathrm{F}(1,33)=4.21, \mathrm{p}<.05]$. The interaction was not statistically significant $[F(1,33)=1.77, p>.1]$.

In summary, it is clear that the two groups which had been reinforced during Phase 2 for Lever B responses showed a significant recovery of responding on Lever A during Phase 3. The recovery was not affected by whether there had been one or two levers reinforced during Phase 1.

\section{Discussion}

The importance of this experiment centers on the recovery of responding in Phase 3. The discriminative cue hypothesis would argue that the recovery demonstrated by the standard alternative response group in Phase 3 was due to Lever B reinforcement serving as a discriminative cue for extinction conditions on Lever A. Since the concurrent alternative response group was exposed to Lever B reinforcement prior to the introduction of extinction conditions on Lever A and therefore Lever B reinforcement could not have served as a discriminative cue for extinction, this group should have shown no recovery. The Phase 3 data obviously require that the discriminative cue hypothesis be rejected, since there was no difference between the two alternative response groups in the amount of Lever A recovery. If anything, the 
concurrent alternative response group responded slightly, but not significantly more.

It would seem that high frequency reinforcement of alternative behavior during the first half of an extinction phase acts akin to procedures which physically prevent the animal from emitting the response programmed for extinction. The response to be extinguished is quickly and almost completely supplanted by the new reinforced alternative response. Thus it never sufficiently encounters extinction conditions, i.e., repeated emission of a response followed by nonreinforcement.

\section{REFERENCES}

Azrin, N. H., \& Holz. W. C. Punishment. In W. K. Honig (Ed.), Operant behavior: Areas of research and application. New York: Appleton-Century-Crofts. 1966.

Boe. E. E., \& С Сurch, R. M. Permanent effect of punishment during extinction. Journal of Comparative and Physiological Psychology. 1967, 63, 486-492.
Enkema, S., Slavin, R., Spaeth, C., \& Neuringer, A. Extinction in the presence of free food. Psychonomic Science, 1972, 26. 267-269.

Estes. W. K. An experimental study of punishment. Psychological Monographs, 1944. 57. Whole No. 263.

Ferster. C. B.. \& Skinner, B. F. Schedules of reinforcement. New York: Appleton-Century-Crofts. 1957.

Leitenberg. H., Rawson. R. A., \& Bath, K. Reinforcement of competing behavior during extinction. Science. 1970, 169. 301-303.

Leitenberg, H., Rawson, R. A., \& Mulick, J. A. Extinction and reinforcement of alternative behavior. Joumal of Comparative and Physiological Psychology. 1975. 88. 640-652.

Rawson, R. A.. \& LeItenBerg, H. Reinforced alternative behavior during punishment and extinction with rats. Journal of Comparative and Physiological Psychology, 1973, 85, 593-600.

SKINNER. B. F. The behavior of organisms: An experimental analysis. New York: Appleton-Century-Crofts. 1938.

SkINNER, B. F. Are theories of learning necessary? Psychological Review. 1950, 57, 193-216.

Winer. B. J. Statistical principles in experimental design. New York: McGraw-Hill. 1971.

(Received for publication August 16, 1976; revision accepted August 5. 1977.) 